\title{
Significances of OMV and Extracellular Vesicle Proteomics
}

Tiwari V*, Solanki V, Roy R, Biswas D and Tiwari M

Department of Biochemistry, Central University of Rajasthan, Ajmer, Rajasthan, 305817, India

*Corresponding author: Tiwari V, Department of Biochemistry, Central University of Rajasthan, Ajmer, Rajasthan, 305817, India, Tel: +91-1463-238652; Fax: +91-1463-238722; E-mail: vishvanath7@yahoo.co.in

Received date: Oct 30, 2016; Accepted date: Nov 28, 2016; Published date: Dec 05, 2016

Copyright: (c) 2016 Tiwari V, et al. This is an open-access article distributed under the terms of the Creative Commons Attribution License, which permits unrestricted use, distribution, and reproduction in any medium, provided the original author and source are credited.

\begin{abstract}
Outer Membrane Vesicle (OMV) proteome has been involved into the pathogenesis of diseases and resistance of microorganisms against a number of antibiotics, mechanism of action of probiotics and host-pathogen interaction etc. We have enlightened the role played by extracellular vesicles proteomics in the pathogenesis of different diseases related to human. Isolation succeeded by purification of ample amount of OMV from biological samples, is one of the most important steps for further proteome related analysis. With the development of both labelled and label-free methods used in proteomics, significant progress has been made in previous years in membrane proteomics. Hence, it is important to review the biological significance of proteins found in the OMV fractions using membrane proteomics approach. We have also explained methods used for isolation, purification and quantification of OMV. In the present review, it can be concluded that proteomic study of outer membrane and extracellular vesicles has now gained priority for the detailed study of disease pathogenesis, drug resistance, vaccine development, cell signalling etc.
\end{abstract}

Keywords: Membrane proteomics; Outer membrane vesicles proteomics; Extracellular vesicle proteomics, Proteomics; Methods for OMV purification

\section{Introduction}

Microorganisms communicate among themselves and with their surrounding environment via membrane vesicles found in their Outer Membrane Vesicle (OMV) for trafficking bacterial cell signalling molecules to target cells around them. These molecules include nucleic acids, proteins, some endotoxins and allied virulence factors. Gramnegative bacteria are known to constitutively secrete OMVs in their extracellular space. Their production is the response of bacterial stress and crucial for taking up nutrients from the environment, carrying endotoxic lipopolysaccharide, biofilm formation, quorum sensing, genetic transformations, and pathogenesis. They facilitate enzymes to reach their targets in a concentrated and protected irrespective of the distance from its source. Adhesions, toxins, and immune modulatory compounds containing OM vesicles that are synthesized naturally by pathogenic bacteria, facilitates the spread of pathogenesis of bacteria by enhancing its binding and invasion, resulting in cytotoxicity, and modifying immune response of host [1]. OMVs are also reported to behave as secretion system for factors related to virulence and toxins inside a host, facilitate survival in the inter-species environmental competition, intra- and inter-species communication, secretion of proteins which are misfolded and aggregated (a novel stress response mechanism) etc.[2]. Both pathogenic and non-pathogenic species of Gram-negative bacteria are known to release vesicles [3-6], including Escherichia coli [7,8], Borrelia burgdorferi [9], Neisseria spp. [10,11], Bacteroides (including Porphyromonas) spp. [12-14], Shigella spp. [15,16], Helicobacter pylori [17], Salmonella spp. [18,19], Brucella melitensis [20], Campylobacter jejuni [21,22], Xenorhabdus nematophilus [23], Vibrio spp. [24,25] Actinobacillus actinomycetemcomitans [26], and Pseudomonas aeruginosa [27]. Studies related to the vesicles released by various origins of bacteria suggest that these vesicles facilitate interactions between prokaryotic or eukaryotic cells with and bacteria. Artificial outer membrane vesicles have also been designed to study the role of OMVs such as OMV of Pseudomonas aeruginosa [28]. OMVs reduce the toxic compound levels like toluene resulting in the release of attacking phage thereby protecting the bacteria $[29,30]$. OMVs based vaccines are capable of conferring immunity against the various genotypes of Acellular pertussis [31], Neisseria meningitis [32], Pseudomonas aeroginosa [33], Bordetella parapertussis and Bordetella pertussis infection [34]. $\mathrm{OMV}$ and detergent extracted outer membrane vesicles (DOMV) are also used as vaccine delivery system [35-39]. In this review, we have discussed the significance of the different methods used for isolation and characterization of the outer-membrane and extra-cellular proteome.

\section{Membrane vesicles}

Prokaryotes, eukaryotes (archaea [40,41], Gram-negative and Gram-positive bacteria [42-45], fungi [45-46] and parasites [50,51] produce the spherical, membranous vesicles from the outer cell surface. Archaeal membrane vesicles (diameter 90-230 nm) contain Slayer proteins and membrane lipids, which has been obtained from the surface of archaeal cell [41,52]. Archaea MVs produces both toxin [52] and non-toxin [53] releasing vesicles. Microbial vesicles obtained from parasites and fungi; include two types of vesicles [48,54]. Exosomes (diameter of 50-100 nm) are known to be produced from intracellular compartments and ectosomes, which are shed from plasma membrane [55]. Exosomes are typically homogeneously shaped vesicles produced during exocytosis of multivesicular bodies within the cell, while ectosomes are ubiquitous in nature that assembled at and released out from plasma membrane of cell [56]. Microvesicles (100-1,000 nm in diameter), similar to OMV are produced from plasma membrane and endosomal membrane compartment by hematopoietic cells, epithelial cells, and cells of tumor. Proteins, mRNAs, microRNAs, and lipids make up the bilayer proteolipids named as extracellular vesicles [55]. It 
is also important to note that Extracellular Vesicles (EVs) (similar to bacterial OMV in component), which are released by various types of mammalian cells, facilitates communication between different cells [57]. Bacterial EV are proposed to contribute to intercellular communication, bacterial survival and human pathogenesis as a novel secretion system [58]. EVs can transport proteins, nucleic acids like mRNAs and microRNAs [57]; transport membrane components including receptors [59]; and protect the stuffs they are carrying from getting degraded by many enzymes present in the extracellular region [57]. Quantitative proteomic study of $S$. typhimurium revealed that membrane vesicles released by bacteria are modulated by the envelope proteins [60]. The location of membrane vesicle formation is the protein enriched regions of envelope characterized by temporary decrease in OM-PG and/ OM-PG-IM interconnections density [60] or slight modulation in LPS structure [61] leads to the change in MV formation. High conservation of protein domains enhances the interconnections of OM-PG-IM and OM-PG envelope among different Gram-negative bacteria [60] portray that these connections play a crucial role in the vesicle release pathway of bacteria. Although fungi, parasites, and archaea are not related taxonomically but several basic features of vesicle production are same perhaps upon which lots of other microorganisms have adapted additional mechanisms. Archaeal MVs are synthesized and secreted by pinching the cell surface off, a phenomenon reminiscent of eukaryotic SMVs and bacterial MVs.

\section{Formation of Outer membrane vesicles}

Outer Membrane Vesicles (OMV) biogenesis without disrupting the viability of bacteria still remains intangible. Various vesiculation mechanism theories based on genetic and biochemical data have been studied [62]. The ubiquitous process of vesiculation helps in the survival of Gram-negative bacteria in different conditions of environments [3]. Naturally produced membrane vesicles are very distinct, closed blebs of outer membrane released by budding cells, rather due to cell lysis or death [14,62-64]. The utmost rate of membrane vesicle formation takes place at the end of log phase and is plentiful at the sites of cell division, as explained for $B$. melitensis, $E$. coli and Vibrio cholera [8,20-24]. The outer membranes of Gramnegative bacteria consist of proteins, asymmetric lipid distribution, phospholipids, lipoproteins and lipopolysaccharide (LPS). The periplasm comprises of the peptidoglycan layer, occupant housekeeping enzymes and proteins, and temporary intermediates of secretory pathways. Native OMVs contain only lipids and protein of the outer membrane as well as periplasm but do not contain components of cytoplasm and inner membrane. This was confirmed from density gradient-purified OMV analysis [62]. To understand the mechanism of vesicle formation and release, deletion of $y f g L$ gene, leads to remarkable decline in formation and release of OMV and in an adherent-invasive strain of E. coli $\mathrm{K} 12$ and E. coli (AIEC) [65]. A lipoprotein is encoded by $y f g L$ gene, which plays role in the formation and/or degradation of peptidoglycan [66]. yfgL resulted in rise of the peptidoglycan production and decrease in the lytic transglycosylase synthesis which in turn reduce the turgor pressure on outer membrane of cell [65]. This experiment revealed that in the synthesis of the
OMVs, LPS structure plays an important role directly and indirectly. In general, non-pathogenic bacteria produce less number of vesicles than their pathogenic counterparts $[67,68]$. PAO1 strain of $P$. aeruginosa, has two types of $O$-antigen side chain, produce the highly charged and long "B-band" form vesicles $[27,69,70]$. This strain also increases the vesicle formation with the increment in B-band LPS under the different condition (oxygen stress) [71]. Salmonella and $P$. aeruginosa mutants also show the increase in vesicle production in which LPS O-antigen side chain is missing $[72,73]$ whereas mutation in core of LPS result in decreased OMP expression [73-75]. Some recent studies demonstrated that various pathways that manage different environmental stresses that are faced by a pathogen inside host body influence activation of pathways involved in OMV formation of $P$. aeruginosa. These stresses increase the OMV production, which does not depend on modulators of OMV formation identified from previous studies, such as PQS, MucD and, AlgU, homolog modulators of OMV synthesis in E. coli. It was also revealed that B-band instead of A-band LPS was responsible for OMV biogenesis induced by oxidative-stress. However, the process by which increased OMV formation helps $P$. aeruginosa to adjust with stressful conditions inside host body and survival, is yet to be studied [76].

\section{Proteomic analysis of OMV and EVs}

Outer membrane related proteins that are exposed on the surface and extracellular proteins, play a role in adhesion, entry, transport of nutrients, toxicity, suppression of host cell immune system and also resistance to the antibiotic. Consequently, identifying and characterizing the proteins associated with OMV can improve the diagnosis of diseases and result in the development of new vaccine and drug targets. Proteomics is considered as a major technique to study membrane proteomics in detail under different diverse conditions [77]. With the development of proteomic tools [77-80], considerable progress has been done in recent years in the area of membrane proteomics. Various methods that are employed in the membrane and differential proteomics are unlabelled methods, labelled method and most recent label free quantification methods $[79,80]$. Isotope labels can be integrated into peptides metabolically, chemically or enzymatically. In label free proteomics the mass spectrometry reveals the mass differences and their proteomic quantifications can be done by analyze their respective signal intensities. Every isolation and purification method has its own merits and demerits and they are paired to each other [79]. Based on the above introduction, it is also important to explain the significance and use of OMV and extracellular vesicle proteomics. Nowadays, numerous studies are being performed on proteomic analysis of bacterial OMVs. Recent progress in the studies of Gram-negative bacterial extracellular vesicles implies that OMVs may function as intercellular communicasomes in bacteria-bacteria and bacteria-host interactions [81]. Some studies show that $70-80 \%$ OM- associated proteins make up the OMVs [82]. Hence, the surface proteomes have been analyzed to get a better understanding of the mechanism related to virulence, drug resistance, biofilm formation and development of new drug and vaccine targets. Different steps of isolation, purification, quantification of OMV and its significances have been outlined in Figure 1. 


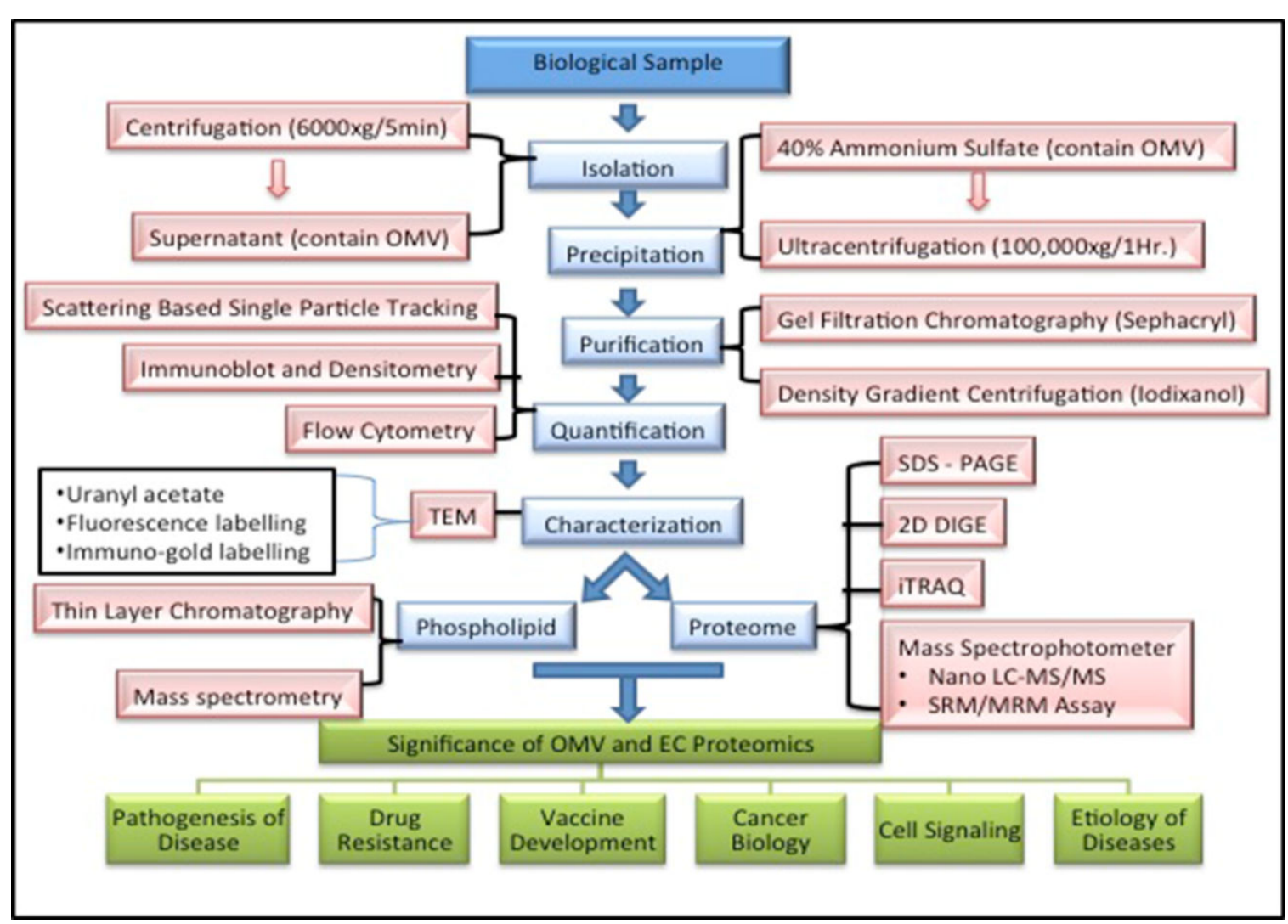

Figure 1: Outline of different steps of isolation, purification and quantification of OMV and significances of OMV.

\section{Isolation, purification and quantification of OMV and EV}

Before performing the proteomics, it is important to review the methods for the isolation and purification of the OMV. Methods used for the isolation of the OMV and EV include ultracentrifugation and precipitation [2]. The isolation of OMV starts from culture supernatant after removal of cultured bacteria by centrifugation at $6,000 \times \mathrm{g}$ for 5 min. Supernatant was filtered via $0.22 \mu \mathrm{m}$ sterilization vacuum filter (V25) [83]. The non-OMV proteins are removed by filtration using the cut-off of $50-100 \mathrm{kDa}$ membranes before ultracentrifugation. Precipitation of the OMV has been performed using ammonium sulfate. A particular concentration of ammonium sulfate is required for the isolation OMV therefore care must be taken to select the concentration of the ammonium sulfate. For example in $P$. gingivalis, OMV proteins are precipitated by $40 \%$ saturation of ammonium sulfate $[12,14]$ followed by the isolation of non-OMV proteins at $70 \%$ saturation [84]. This shows that a proper concentration of ammonium sulfate should be resolute carefully and imprecise binding of extracellular proteins to ammonium sulfate-precipitated OMV is exceedingly feasible [1]. The increase in protease during concentration step may results in OMV associated protein degradation. Hence, addition of corresponding inhibitor into the media prior to the concentration step is required to prevent the degradation. The precipitated and centrifuged OMV should then be reconstituted by dialysis against suitable buffer before further investigations. Both these isolation methods are unable to isolate OMV from the extra cellular matrixes such as large protein complex and aggregates. These impurities are removed by the density gradient centrifugation and gel filtration. In the OMV, lipid content is more than secreted proteins and its density is lower than other protein complexes, hence OMV, move around to lighter fractions during the iodixanol density gradient centrifugation [85]. Gel filtration is also employed for the purification of the isolated OMV using Sephacryl S-500 column (i.e., $N$. meningitidis OMV) [86], Sephacryl S-300 (Meiothermus ruber OMV) [87]. Quantification of OMV can be done by light scattering based single particle tracking [88] and flow cytometric analysis [89]. Bicinchoninic acid assay [90], Lowry [91] and Bradford [92], or by image analysis of stained or immune-detected gel-separated sample, are also used for OMV component quantification. Proteins extracted OMV can also analyzed by immunoblot and densitometry $[93,94]$. The purified OMV are characterized by TEM microscopy [60] or by gel free and gel-based proteomics method followed by western blotting and mass spectrometry [94]. To achieve the basic TEM images of OMV, negative staining by $2 \%$ uranyl acetate has been performed [88]. Due to excessive cross-linking of the proteins, image background becomes complex and hard to study; therefore, fixation of the specimen has been done by glutaraldehyde and formaldehyde followed by Immunogold labelling to highlight the OMV components $[27,96]$. Examination by fluorescence labeling confirmed the interaction of OMV with other membranes, such as fusion with host-cell membrane before their entry into the eukaryotic cell [97,98]. Phospholipids and fatty acids forming the OMV membrane bilayers are also matter of interest which are first extracted from OMV and then analyzed by thin layer chromatography or mass spectrometry $[99,100]$. 


\section{Significance of OMV proteomics in virulence of pathogens}

Membrane of Gram-negative bacteria comprises of two polysaccharide bilayers, which consist of inner and outer membrane, the periplasm in between. This complicates the process of virulence factor secretion by the bacteria. These pathogens have developed various strategies; of which few are pathogen-specific, so that virulence factors can have admittance to the extracellular environment, especially bloodstream or tissue cells of the host [101]. Due to the cytotoxicity of $A$. baumannii OMV, virulence is induced in the host organism [102]. Kwon et al., reported that some of the proteins associated with OMVs named as chaperonin GroEL, AmpC-betalactamase, 6,7-dimethyl-8-ribityllumazine synthase, and AbOmpA. Among these proteins, several virulence factor linked proteins were recognized: Putative Zn-dependent protease, bacterioferritin, AbOmpA, catalase, putative protease, $\mathrm{Cu} / \mathrm{Zn}$ superoxide dismutase, putative phospholipase A1 precursor, putative serine protease, and ferrichrome-iron receptor. Adaptive and innate immune responses are modulated by interaction of PAMPs, such as lipopolysaccharide, lipoproteins, outer membrane proteins, with PAMPs receptors in the host cells [103,104]. Among the 26 different outer membrane proteins, AbOmpA has been shown the interaction with the TLR-2 along with a modulated immune response in dendritic and epithelial cells $[105,106]$. In host epithelial cells, AbOmpA also facilitates the adhesion and invasion mechanism of the bacteria [107]. Kwon et al., also studied about OMVs as a virulent factor. In doing so, the OMVs in the absence of bacteria were treated with human cell line, which revealed that AbOmpA was present in the cytoplasm, which signifying that outer MVs directly hold multiple diverse virulence factors to host cells. Cytotoxicity assay of OMVs suggests that these virulence factors also alter the physiology of cells (elongation of the cell) [108]. Some previous studies also suggested that 151 proteins of the strain 173 of $A$. actinomycetemcomitans were identified with the help of LC-MS/MS and via OMV proteome investigation and they were found to be involved in virulence related mechanism. Identified protein such as GroEL, LtxA, TdeA, Omp100, TadZ, RcpC, TadE, BilR1, TadG, TadF, TadD, RcpC, RcpA, Pal, OmpA like protein, Omp18/16, and Omp39 are involved in the virulence related mechanism like cytotoxicity, immune-reactivity and/or pro-inflammatory activity, drug targeting, immune evasion and scavenging of iron and nutrients [109].

Proteins that are essential for the virulence of Pseudomonas aeruginosa [110], and that of Helicobacter pylori [111] have been identified through the LC-MS/MS analysis of outer membrane vesicles. Proteomic profiling of OMV of Campylobacter jejuni using high resolution LTQ-orbitrap spectroscopy identified 134 vesicular proteins which have the significant role in the bacterial infection and communication [112]. Virulence role of the OMV can also be understood from the proteomic study of the samples obtained from the septic human or rat serum [113,114]. Proteomic analyses of extracellular proteins in the Acinetobacter baumannii have revealed their role in defense machinery against the macrophagic attack and also in the state of oxidative stress [115]. The proteomic profiling of OMV of Myxococcus xanthus revealed that the OMV is rich in proteins with hydrolytic functions [116]. Proteomic analyses of OMV of Francisella novicida have also revealed its role in the pathogenesis [117]. Quantitative proteomics analyses of heat stressed Clostridium difficile helped to understand its physiological and metabolic functions used during upshift of temperature mimicking pyrexia [118]. Proteomic study of outer membrane of the E. coli revealed over expression FhuE and FhuA, and YbiL that have role in the iron transport [119]. By differential proteomics study, the role of iron in the continued existence of $A$. baumannii resistance strain (ATCC and carbapenem) in human host is revealed [120,121]. Proteome of the human host changes during Acinetobacter baumannii infection. Soares et al, found that the alterations in the plasma proteome using DIGE based differential proteomic analysis of the host infection with $A$. baumannii as compared to controls [122]. FhuE receptor, ferric acinetobactin receptor, ferrienterchelin receptor and ferric siderophore receptor have been played a role in iron transport in the host, identified in the membrane fraction of $A$. baumannii using DIGE based proteomics tool. Wurpal et al., studied about EDTA-heat induced outer MV biogenesis coupled with proteolytic treatment like carbonate extraction, cell lysis, ultracentrifugation and 2D-DIGE succeeded by MS/MS analysis of different uro-pathogenic E. coli (UPEC) strains. This UPEC surface-exposed proteome analysis demonstrated the constitutive proteins along with the various virulence related proteins [82]. Current MS-based high-throughput proteomic analyses of Gram-negative bacterial OMVs have identified thousands of vesicular proteins and provided clues to reveal the biogenesis and pathophysiological functions of Gram-negative bacterial OMVs [81].

\section{Significance of OMV proteomics in the study of drug resistance and biofilm formation}

Outer membrane act as a barrier between organism and host, hence the drugs have to cross the membrane to exert its action. Outer membrane vesicles are also known to possess chromosomal encoded $\beta$-lactamases which causes the degradation of extracellular $\beta$-lactam [123]. All Gram negative bacteria have been synthesized such as OM vesicles by which they facilitate biofilm formation and many biological functions namely virulence factors transportation and antibiotics resistance $[60,124,125]$. Bacterial mobility is highly conserved in all Xanthomonas, and $S$. maltophilia species. Some recent studies related to OMV associated virulence factor Ax21 was found in S. maltophilia $[126,127]$ while in Xanthomonas oryzae was found to contain the same factor (named Omp1x) [128] which play a role in virulence and biofilm formation [129]. In Xanthomonas pv. oryzicola this protein expression is controlled by DSF quorum sensing system [130]. The Diffusible Signal Factor (DSF) has a major importance in regulating biofilm production as well as cell-cell communication in $S$. maltophilia $[131,132]$. This factor system is also known to involve in cocolonization of the pathogens like $B$. cenocepacia and $P$. aeruginosa [131] in host lungs epithelial cells causing cystic fibrosis [133]. Devos et al., measured the OMV mediated protein secretion of the homologs of $\mathrm{A} \times 21$ in the presence of BDSF, imipenem, PDSF and diffusible signal factors via targeted and label-free LC Multiple Reaction Monitoring technique. The results indicate large quantities of $A \times 21$ protein formation and packaging in OMVs that depicts the role played by it in the biofilm production and antibiotic resistance. Utilizing proteomic analysis, membrane vesicles of Staphylococcus aureus has been shown to have role in antibiotic resistance and pathology [43]. Minami et al., the changes in proteomic profiles of DRM fractions during cold acclimation using 2D-gel electrophoresis and mass analysis [134]. RND multidrug efflux pump membrane fusion proteins have been identified in the $P$. aeruginosa using membrane proteomics approach [86,106,135-137]. Proteomic analyses of A. baumannii explain that histidine metabolism also have major role in the biofilm formation [138]. Outer membrane comparative proteomic analysis of $P$. aeruginosa showed resistance against the ampicillin, kanamycin, and tetracycline antibiotics [139]. Production of vesicles is also influenced by antibiotics like mitomycin $\mathrm{C}$, an inducer of Shiga toxin, used for 
treating Shigella dysenteriae. This increases the OMV size, toxicity and production [15] while antibiotics like norfloxacin, ciprofloxacin, fosfomycin, etc. are not responsible for altering OMV production or toxicity. Carbapenem resistant $A$. baumannii has been already studied using outer membrane proteomics between wild type and resistance strain [108, 140-142]. Membrane proteomics result also showed that $A$. baumannii displays a vigorous and adaptable metabolism [143,144]. Similarly, differential high-end isoelectric point proteome analysis of Acinetobacter radioresistens reveals that with the help of aromatic compounds, we can induce the envelope stress responses [145]. Using differential quantitative proteomic analysis of OMVs from multidrugresistant $A$. baumannii, Yun et al, reported that carbapenem suppress outer membrane proteins expression and increases the expression of resistance nodulation cell division transporters and protein kinases [146]. Using similar approach, Lee et al., explain the mechanism of hetero-resistance mechanism induced by imipenem in the multi-drug resistant $A$. baumannii [147]. Biofilm formation is one of the important reasons for the persistence of $A$. baumannii on the surface of host lung epithelial cells. Cabral et al., did the differential proteomics of multi-drug resistant Acinetobacter cultured in three diverse conditions (exponential, late stationary phase and biofilms stage) and they also examine the effects of salicylate on the biofilm formation which is a biofilm inhibitory compound. This multiple approach strategy explained unique lifestyle of $A$. baumannii, which are involved in both virulence and biofilms formation [148]. Differential proteomics of the Porphyromonas gingivalis, Treponema denticola suggest synergestic relationship in the polymicrobial biofilms formation [149].

\section{Extracellular vesicles (EV) proteomics in the study of human diseases}

EVs act as vehicles for intercellular communication and host manipulation [150]. Cancer has traditionally been considered as a human disease resulting from gene mutations but new finding suggest that extracellular vesicles which are derived from tumour can carry polypeptides, polysaccharides, DNA and RNA causative of cancer progression [151]. Intake of the tumor derived extracellular vesicles (EVs) by non-tumor cells can be converted into cancerous cell [152]. EV released by cancer cells also acts against the stress response [93]. Bioengineering of the membrane vesicles for the delivery of the siRNA into the cancer cell and used in the cancer therapy [153]. Exosomes secretion has been found to be key feature for the malignancy of the different stages of the cancer growth and development [59]. The OMVs released from Helicobacter pylori increases the chance of development of gastric cancer [154]. Biomarkers have been identified form the limited breast cancer tissue by membrane proteomics using SRM/MRM proteomic analysis in combination with iTRAQ shotgun analysis [155]. Proteomic analysis of purified MVs [156] from colorectal cancer cells has been done using SDS PAGE and nano LCMS/MS analysis [157]. Tumor-derived MVs also have a role in intercellular communication known as extracellular organelles communicasomes [157]. The mitochondrial and endoplasmic reticulum fraction of breast cancer cell lines ZR-75-1 cells and MDAMB-231 cells treatment with Dox-TRAIL identified new differentially abundant proteins using the iTRAQ labelling coupled with multidimensional LC-MS/MS [158]. Proteomic analysis also explained that serum glycoprotein can be a reliable biomolecule for the identification of biomarkers in the pancreatic cancer [159]. Extracellular vesicles are shed to the extracellular region by most eukaryotic and prokaryotic cells but recently it has been reported in
Gram-positive bacteria, mycobacteria and fungi [160]. EV proteomes reflects developmental phases of Bacillus subtilis where it shows the formation of EV during sporulation is strongly supported by delineation of protein content that differs from proteome of EV formed by vegetative spores [58].

\section{Significance of OMV in the development of probiotics}

Probiotics are the microorganisms that provide health benefits when consume. E. coli Nissle 1917 (EcN) is the well-known probiotic that is used in treatment of intestinal disease. This enhances the useful microbiota and the homeostasis of the gastro-intestines. Using the LCMS/MS analysis, Aguilera et al., identified 18 different proteins that have been found to be strain specific and others are outer membrane vesicles of pathogens. These outer membrane vesicles shows binding with the host and increase the beneficial and positive effects on the host intestinal function [161]. Moreover, OMVs are known to be strong immunomodulatory, so they can also be used as potent pathogen-free vaccines after modifying its immunogenic contents. Proteomic analysis of OMV has enabled researchers to identify new vaccine targets against various pathogens by increasing host immune responses. This includes neonatal meningitis E. coli (NMEC) [136], group A Streptococcus [160] and Neisseria meningitidis serogroup B [161].

\section{Conclusion and Future Perspective}

OMV proteomics gives a better and clear understanding of the role of various host micro-environments that contribute to the pathogen survival. Because of their role in communication between species, interaction of host and pathogen and adaptability to various environment conditions, OMV is potential source for the vaccine development. Isolation followed by purification of sufficient quantity of outer membrane vesicles from biological samples is the most crucial step for subsequent investigations such as quantitation and characterization. With the advancement in the proteomic methods significant progress has been made in the discipline of extracellular proteomics and OMV. Therefore, outer membrane and extra cellular proteomics has now become much more apparent as methods of choice for studying the pathogenesis of diseases, drug resistance, vaccine development, cancer biology, cell signalling etc. Various approaches [162-163] have been tried to develop effective drug against the pathogen and OMV proteomics can pay a significant role in this development. Study of these vesicles proteomics will also help in the better understanding of etiology of the diseases and hence help in the development of effective drug.

\section{Acknowledgement}

VT (for Grant) and RR (for JRF fellowship) would like to thanks SERB, DST, and India for Start Up grant (SB/YS/LS-07/2014). MT would like to thanks Central University of Rajasthan for the Ph. D. fellowship. VS and DB would like to thanks UGC for the JRF fellowship.

\section{Conflict of Interest}

The authors have declared that no competing interests exist. 


\section{References}

1. Kulp A, Kuehn MJ (2010) Biological functions and biogenesis of secreted bacterial outer membrane vesicles. Ann Rev Microbiol 64: 163-184.

2. Klimentova J, Stulik J (2015) Methods of isolation and purification of outer membrane vesicles from gram-negative bacteria. Microbiol Res 170: $1-9$.

3. Beveridge TJ (1999) Structures of Gram-negative cell walls and their derived membrane vesicles. J Bacteriol 18: 4725-4733.

4. Kadurugamuwa JL, Beveridge TJ (1997) Natural release of virulence factors in membrane vesicles by Pseudomonas aeruginosa and the effect of aminoglycoside antibiotics on their release. J Antimicrob Chemother 40: 615-621.

5. Li Z, Clarke A, Beveridge TJ (1998) Gram-negative bacteria produce membrane vesicles which are capable of killing other bacteria. J Bacteriol 180: 5478-5483.

6. Mayrand D, Grenier D (1989) Biological activities of outer membrane vesicles. Can J Microbiol 35: 607-613.

7. Gankema H, Wensink J, Guinee PA, Jansen WH, Witholt B (1980) Some characteristics of the outer membrane material released by growing enterotoxigenic Escherichia coli. Infect Immun 29: 704-713.

8. Hoekstra D, van der Laan JW, de Leij L, Witholt B (1976) Release of outer membrane fragments from normally growing Escherichia coli. Biochimica et Biophysica Acta 455: 889-899.

9. Shoberg RJ, Thomas DD (1993) Specific adherence of Borrelia burgdorferi extracellular vesicles to human endothelial cells in culture. Infect Immun 61: 3892-3900.

10. Devoe IW, Gilchrist JE (1973) Release of endotoxin in the form of cell wall blebs during in vitro growth of Neisseria meningitidis. J Exp Med 138: 1156-1167.

11. Dorward DW, Garon CF (1989) DNA-binding proteins in cells and membrane blebs of Neisseria gonorrhoeae. J Bacteriol 171: 4196-4201.

12. Grenier D, Mayrand D (1987) Functional characterization of extracellular vesicles produced by Bacteroides gingivalis. Infect Immun 55: 111-117.

13. Mayrand D, Holt SC (1988) Biology of asaccharolytic blackpigmented Bacteroides species. Microbiol Rev 52: 134-152.

14. Zhou L, Srisatjaluk R, Justus DE, Doyle RJ (1998) On the origin of membrane vesicles in gram-negative bacteria. FEMS Microbiol Lett 163: 223-228.

15. Dutta S, Iida K, Takade A, Meno Y, Nair GB, et al. (2004) Release of Shiga toxin by membrane vesicles in Shigella dysenteriae serotype 1 strains and in vitro effects of antimicrobials on toxin production and release. Microbiol Immunol 48: 965-969.

16. Kadurugamuwa JL, Beveridge TJ (1999) Membrane vesicles derived from Pseudomonas aeruginosa and Shigella flexneri can be integrated into the surfaces of other Gram-negative bacteria. Microbiology 145: 2051-2060.

17. Fiocca R, Necchi V, Sommi P, Ricci V, Telford J, et al. (1999) Release of Helicobacter pylori vacuolating cytotoxin by both a specific secretion pathway and budding of outer membrane vesicles. Uptake of released toxin and vesicles by gastric epithelium. J Pathol 188: 220-226.

18. Vesy CJ, Kitchens RL, Wolfbauer G, Albers JJ, Munford RS (2000) Lipopolysaccharide-binding protein and phospholipid transfer protein release lipopolysaccharides from gram-negative bacterial membranes. Infect Immun 68: 2410-2417.

19. Wai SN, Lindmark B, Soderblom T, Takade A, Westermark M, et al. (2003) Vesicle-mediated export and assembly of pore-forming oligomers of the enterobacterial ClyA cytotoxin. Cell 115: 25-35.

20. Gamazo C, Moriyon I (1987) Release of outer membrane fragments by exponentially growing Brucella melitensis cells. Infect Immun 55: 609-615.

21. Blaser MJ, Hopkins JA, Berka RM, Vasil ML, Wang WL (1983) Identification and characterization of Campylobacter jejuni outer membrane proteins. Infect Immun 42: 276-284.

22. Logan SM, Trust TJ (1982) Outer membrane characteristics of Campylobacter jejuni. Infect Immun 38: 898-906.
23. Khandelwal P, Banerjee-Bhatnagar N (2003) Insecticidal activity associated with the outer membrane vesicles of Xenorhabdus nematophilus. Appl Environ Microbiol 69: 2032-2037.

24. Chatterjee SN, Das J (1967) Electron microscopic observations on the excretion of cell-wall material by Vibrio cholerae. J Gen Microbiol 49: $1-11$.

25. Kondo K, Takade A, Amako K (1993) Release of the outer membrane vesicles from Vibrio cholerae and Vibrio parahaemolyticus. Microbiol Immunol 37: 149-152.

26. Nowotny A, Behling UH, Hammond B, Lai CH, Listgarten $\mathrm{M}$, et al. (1982) Release of toxic microvesicles by Actinobacillus actinomycetemcomitans. Infect Immun 37: 151-154.

27. Kadurugamuwa JL, Beveridge TJ (1995) Virulence factors are released from Pseudomonas aeruginosa in association with membrane vesicles during normal growth and exposure to gentamicin: a novel mechanism of enzyme secretion. J Bacteriol 177: 3998-4008.

28. Wessel AK, Liew J, Kwon T, Marcotte EM, Whiteley (2013) M Role of Pseudomonas aeruginosa peptidoglycan-associated outer membrane proteins in vesicle formation. J Bacteriol 195: 213-219.

29. Kobayashi H, Uematsu K, Hirayama H, Horikoshi K (2000) Novel toluene elimination system in a toluene-tolerant microorganism. J Bacteriol 182: 6451-6455.

30. Loeb MR, Kilner J (1978) Release of a special fraction of the outer membrane from both growing and phage T4-infected Escherichia coli. Biochim Biophys Acta 514: 117-127.

31. Gaillard ME, Bottero D, Errea A, Ormazabal M, Zurita ME, et al. (2014) Acellular pertussis vaccine based on outer membrane vesicles capable of conferring both long-lasting immunity and protection against different strain genotypes. Vaccine 32: 931-937.

32. Pinto VB, Burden R, Wagner A, Moran EE, Lee CH (2013) The development of an experimental multiple serogroups vaccine for Neisseria meningitidis. PloS ONE 8: e79304.

33. Zhao K, Deng X, He C, Yue B, Wu M (2013) Pseudomonas aeruginosa outer membrane vesicles modulate host immune responses by targeting the Toll-like receptor 4 signaling pathway. Infect Immun 81: 4509-4518.

34. Bottero D, Gaillard ME, Errea A, Moreno G, Zurita E, et al. (2013) Outer membrane vesicles derived from Bordetella parapertussis as an acellular vaccine against Bordetella parapertussis and Bordetella pertussis infection. Vaccine 31: 5262-5268.

35. Chen DJ, Osterrieder N, Metzger SM, Buckles E, Doody AM, et al. (2010) Delivery of foreign antigens by engineered OMV vaccines. Proc Natl Acad Sci USA 107: 3099-3104.

36. Kim SH, Kim KS, Lee SR, Kim E, Kim MS, et al. (2009) Structural modifications of outer membrane vesicles to refine them as vaccine delivery vehicles. Biochim Biophys Acta 1788: 2150-2159.

37. Bartolini E, Ianni E, Frigimelica E, Petracca R, Galli G, et al. (2013) Recombinant outer membrane vesicles carrying Chlamydia muridarum HtrA induce antibodies that neutralize chlamydial infection in vitro. J Extracell Vesicles 2: 2018.

38. Tunheim G, Arnemo M, Naess LM, Fjeldheim AK, Nome L, et al. (2013) Preclinical immunogenicity and functional activity studies of an $\mathrm{A}+\mathrm{W}$ meningococcal OMV(OMV) vaccine and comparisons with existing meningococcal conjugate and polysaccharide vaccines. Vaccine 31: 6097-6106.

39. Norheim G, Tunheim G, Naess LM, Kristiansen PA, Caugant DA, et al. (2012) An OMV vaccine for prevention of serogroup A and W-135 meningococcal disease in the African meningitis belt. Scand J Immunol 76: 99-107.

40. Ellen AF, Zolghadr B, Driessen AM, Albers SV (2010) Shaping the archaeal cell envelope. Archaea 2010: 1-13.

41. Ellen AF (2008) Proteomic analysis of secreted membrane vesicles of archaeal Sulfolobus species reveals the presence of endosome sorting complex components. Extremophiles 13: 67-79. 
42. Dorward DW, Garon CF (1990) DNA is packaged within membrane derived vesicles of gram-negative but not gram-positive bacteria. App Environ Microbiol 56: 1960-1962.

43. Lee EY, Choi DY, Kim DK, Kim JW, Park JO, et al. (2009) Gram-positive bacteria produce membrane vesicles: proteomics-based characterization of Staphylococcus aureus-derived membrane vesicles. Proteomics 9: 5425-5436.

44. McBroom AJ (2005) Outer membrane vesicles. In Finlay BB (ed.) EcoSalEscherichia coli and Salmonella: cellular and molecular biology. ASM Press, Washington, DC.

45. Rivera J, Cordero RJ, Nakouzi AS, Frases S, Nicola A, et al. (2010) Bacillus anthracis produces membrane-derived vesicles containing biologically active toxins. Proc Natl Acad Sci USA 107: 19002-19007.

46. Albuquerque PC, Nakayasu ES, Rodrigues ML, Frases S, Casadevall A, et al. (2008) Vesicular transport in Histoplasma capsulatum: an effective mechanism for trans-cell wall transfer of proteins and lipids in ascomycetes. Cell Microbiol 10: 1695-1710.

47. Nosanchuk JD, Nimrichter L, Casadevall A, Rodrigues ML (2008) A role for vesicular transport of macromolecules across cell walls in fungal pathogenesis. Commun Integr Biol 1: 37-39.

48. Oliveira DL, Nakayasu ES, Joffe LS, Guimaraes AJ, Sobreira TJ, et al (2010) Biogenesis of extracellular vesicles in yeast. Commun Integr Biol 3: 533-535.

49. Oliveira DL, Nakayasu ES, Joffe LS, Guimaraes AJ, Sobreira TJ, et al. (2010) Characterization of yeast extracellular vesicles: Evidence for the participation of different pathways of cellular traffic in vesicle biogenesis. PloS ONE 5: e11113.

50. Silverman JM, Chan SK, Robinson DP, Dwyer DM, Nandan D, et al. (2008) Proteomic analysis of the secretome of Leishmania donovani. Genome biology 9: R35

51. Silverman JM (2010) An exosome-based secretion pathway is responsible for protein export from Leishmania and communication with macrophages. J Cell Sci 123: 842-852.

52. Prangishvili D, Holz I, Stieger E, Nickell S, Kristjansson JK, et al. (2000) Sulfolobicins. specific proteinaceous toxins produced by strains of the extremely thermophilic archaeal genus Sulfolobus. J Bacteriol 182: 2985-2988.

53. Rachel R, Wyschkony I, Riehl S, Huber H (2002) The ultrastructure of Ignicoccus: evidence for a novel outer membrane and for intracellular vesicle budding in an archaeon. Archaea 1: 9-18.

54. Gyorgy B, Szabo TG, Pasztoi M, Pal Z, Misjak P, et al. (2011) Membrane vesicles: current state-of-the-art: emerging role of extracellular vesicles. Cell Mol Life Sci 68: 2667-88.

55. Choi DS, Kim DK, Kim YK, Gho YS (2013) Proteomics, transcriptomics and lipidomics of exosomes and ectosomes. Proteomics 13: 1554-1571.

56. Cocucci E, Meldolesi J (2015) Ectosomes and exosomes: shedding the confusion between extracellular vesicles. Trends Cell Biol 25: 364-372.

57. Katsuda T, Kosaka N, Ochiya T (2014) The roles of extracellular vesicles in cancer biology: toward the development of novel cancer biomarkers. Proteomics 14: 412-425.

58. Kim Y, Edwards N, Fenselau C (2016) Extracellular vesicle proteomes reflect developmental phases of Bacillus subtilis. Clin Proteomics 13: 6 .

59. Marleau AM, Chen CS, Joyce JA, Tullis RH (2012) Exosome removal as a therapeutic adjuvant in cancer. J Transl Med 10: 134.

60. Deatherage BL, Lara JC, Bergsbaken T, Rassoulian Barrett SL, Lara S, et al. (2009) Biogenesis of bacterial membrane vesicles. Mol Microbiol 72: 1395-1407.

61. Munford RS, Varley AW (2006) Shield as signal: lipopolysaccharides and the evolution of immunity to gram-negative bacteria. PLoS Pathog 2: e67.

62. McBroom A, Kuehn MJ (2005) Outer membrane vesicles In: Curtiss III R (ed.) EcoSal-Escherichia coli and Salmonella: Cellular and molecular biology, ASM Press, Washington, DC.

63. Mug-Opstelten D, Witholt B (1978) Preferential release of new outer membrane fragments by exponentially growing Escherichia coli. Biochim Biophys Acta 508: 287-295.
64. Yaganza ES, Rioux D, Simard M, Arul J, Tweddell RJ (2004) Ultrastructural alterations of Erwinia carotovora subsp. atroseptica caused by treatment with aluminum chloride and sodium metabisulfite. Appl Environ Microbiol 70: 6800-6808.

65. Rolhion N, Barnich N, Claret L, Darfeuille-Michaud A (2005) Strong decrease in invasive ability and OMV release in Crohn's diseaseassociated adherent-invasive Escherichia coli strain LF82 with the yfgL gene deleted. J Bacteriol 187: 2286-2296.

66. Eggert US, Ruiz N, Falcone BV, Branstrom AA, Goldman RC, et al. (2001) Genetic basis for activity differences between vancomycin and glycolipid derivatives of vancomycin. Science 294: 361-364.

67. Lai CH, Listgarten MA, Hammond BF (1981) Comparative ultrastructure of leukotoxic and non-leukotoxic strains of Actinobacillus actinomycetemcomitans. J Periodontal Res 16: 379-389.

68. Wai SN, Takade A, Amako K (1995) The release of outer membrane vesicles from the strains of enterotoxigenic Escherichia coli. Microbiol Immun 39: 451-456.

69. Beveridge TJ, Makin SA, Kadurugamuwa JL, Li Z (1997) Interactions between biofilms and the environment. FEMS Microbiol Rev 20: 291-303.

70. Nguyen TT, Saxena A, Beveridge TJ (2003) Effect of surface lipopolysaccharide on the nature of membrane vesicles liberated from the Gram-negative bacterium Pseudomonas aeruginosa. J Electron Microsc 52: $465-469$.

71. Sabra W, Lunsdorf H, Zeng AP (2003) Alterations in the formation of lipopolysaccharide and membrane vesicles on the surface of Pseudomonas aeruginosa PAO1 under oxygen stress conditions. Microbiology 149: 2789-2795.

72. Meadow PM, Wells PL, Salkinoja-Salonen M, Nurmiaho EL (1978) The effect of lipopolysaccharide composition on the ultrastructure of Pseudomonas aeruginosa. J Gen Microbiol 105: 23-28.

73. Smit J, Kamio Y, Nikaido H (1975) Outer membrane of Salmonella typhimurium: Chemical analysis and freeze fracture studies with lipopolysaccharide mutants. J Bacteriol 124: 942-958.

74. Ames GF, Spudich EN, Nikaido H (1974) Protein composition of the outer membrane of Salmonella typhimurium: effect of lipopolysaccharide mutations. J Bacteriol 117: 406-416.

75. Schnaitman CA, Klena JD (1993) Genetics of lipopolysaccharide biosynthesis in enteric bacteria. Microbiol Rev 57: 655-682.

76. Macdonald IA, Kuehn MJ (2013) Stress-induced OMVproduction by Pseudomonas aeruginosa. J Bacteriol 195: 2971-2981.

77. Tiwari V (2014) Membrane Proteomics has emerged as a Tool to Study Carbapenem Resistance in Acinetobacter baumannii. J Proteomics Bioinform 7: e28.

78. Tiwari V, Tiwari M (2015) Phosphoproteomics as an emerging weapon to develop new antibiotics against carbapenem resistant strain of Acinetobacter baumannii. J Proteomics 112: 336-338.

79. Tiwari V, Tiwari M (2014) Quantitative proteomics to study carbapenem resistance in Acinetobacter baumannii. Front Microbiol 5: 512.

80. Tiwari V (2014) Differential proteomics has emerged as a tool to understand carbapenem resistance in Acinetobacter baumannii. J Integr Omics 4: 1-2.

81. Lee J, Kim OY, Gho YS (2016) Proteomic profiling of Gram-negative bacterial outer membrane vesicles: Current perspectives. Proteomics Clin Appl 10: 897-909.

82. Wurpel DJ, Moriel DG, Totsika M, Easton DM, Schembri MA (2015) Comparative analysis of the uropathogenic Escherichia coli surface proteome by tandem mass-spectrometry of artificially induced outer membrane vesicles. J Proteomics 115: 93-106.

83. Gorringe A, Halliwell D, Matheson M, Reddin K, Finney M, et al. (2005) The development of a meningococcal disease vaccine based on Neisseria lactamica outer membrane vesicles. Vaccine 23: 2210-2213.

84. Oishi S, Miyashita M, Kiso A, Kikuchi Y, Ueda O, et al. (2010) Cellular locations of proteinases and association with vesicles in Porphyromonas gingivalis. Eur J Med Res 1: 397-402. 
85. Graham J, Ford T, Rickwood D (1994) The preparation of subcellular organelles from mouse liver in self-generated gradients of iodixanol. Anal Biochem 220: 367-373.

86. Post DM, Zhang D, Eastvold JS, Teghanemt A, Gibson BW, et al. (2005) Biochemical and functional characterization of membrane blebs purified from Neisseria meningitidis serogroup B. J Biol Chem 280: 38383-38394.

87. Kataoka M, Yamaoka A, Kawasaki K, Shigeri Y, Watanabe K (2014) Extraordinary denaturant tolerance of keratinolytic protease complex assemblies produced by Meiothermus ruber H328. Appl Microbiol Biotechnol 98: 2973-2980.

88. Devos S, Van Oudenhove L, Stremersch S, Van Putte W, De Rycke R, et al. (2015) The effect of imipenem and diffusible signaling factors on the secretion of outer membrane vesicles and associated Ax21 proteins in Stenotrophomonas maltophilia. Front Microbiol 6: 298.

89. Wieser A, Storz E, Liegl G, Peter A, Pritsch M, et al. (2014) Efficient quantification and characterization of bacterial outer membrane derived nano-particles with flow cytometric analysis. Int J Med Microbiol 304: 1032-1037.

90. Brown RE, Jarvis KL, Hyland KJ (1989) Protein measurement using bicinchoninic acid: elimination of interfering substances. Anal Biochem 180: 136-139.

91. Lowry OH, Rosebrough NJ, Farr AL, Randall RJ (1951) Protein measurement with the Folin phenol reagent. J Biol Chem 193: 265-275.

92. Bradford MM (1976) A rapid and sensitive method for the quantitation of microgram quantities of protein utilizing the principle of protein-dye binding. Anal Biochem 72: 248-254.

93. McBroom AJ, Kuehn MJ (2007) Release of outer membrane vesicles by Gram-negative bacteria is a novel envelope stress response. Mol Microbiol 63: 545-558.

94. Manning AJ, Kuehn MJ (2011) Contribution of bacterial outer membrane vesicles to innate bacterial defense. BMC Microbiol 11: 258.

95. Lee EY, Choi DS, Kim KP, Gho YS (2008) Proteomics in gram-negative bacterial outer membrane vesicles. Mass Spectrom Rev 27: 535-555.

96. Duperthuy M, Sjostrom AE, Sabharwal D, Damghani F, Uhlin BE, et al. (2013) Role of the Vibrio cholerae matrix protein Bap1 in cross-resistance to antimicrobial peptides. PLoS pathog 9: e1003620.

97. Galka F, Wai SN, Kusch H, Engelmann S, Hecker M, et al. (2008) Proteomic characterization of the whole secretome of Legionella pneumophila and functional analysis of outer membrane vesicles. Infec Immun 76: 1825-1836.

98. Bomberger JM, Maceachran DP, Coutermarsh BA, Ye S, O'Toole GA, et al. (2009) Long-distance delivery of bacterial virulence factors by Pseudomonas aeruginosa outer membrane vesicles. PLoS Pathog 5 e1000382.

99. Matyash V, Liebisch G, Kurzchalia TV, Shevchenko A, Schwudke D (2008) Lipid extraction by methyl-tert-butyl ether for high-throughput lipidomics. J Lipid Res 49: 1137-1146.

100. Chowdhury C, Jagannadham MV (2013) Virulence factors are released in association with outer membrane vesicles of Pseudomonas syringae pvtomato T1 during normal growth. Biochim Biophys Acta 1834: 231-239.

101. Henderson IR, Navarro-Garcia F, Desvaux M, Fernandez RC, Ala'Aldeen D (2004) Type $\mathrm{V}$ protein secretion pathway: the autotransporter story. Microbiol Mol Bio Rev 68: 692-744.

102. Jin JS, Kwon SO, Moon DC, Gurung M, Lee JH, et al. (2011) Acinetobacter baumannii secretes cytotoxic outer membrane protein A via outer membrane vesicles. PloS ONE 6: e17027.

103. Kuehn MJ, Kesty NC (2005) Bacterial outer membrane vesicles and the host-pathogen interaction. Genes Dev 19: 2645-2655.

104. Bauman SJ, Kuehn MJ (2006) Purification of outer membrane vesicles from Pseudomonas aeruginosa and their activation of an IL-8 response. Microb Infect 8: 2400-2408.

105. Kim SA, Yoo SM, Hyun SH, Choi CH, Yang SY, et al. (2008) Global gene expression patterns and induction of innate immune response in human laryngeal epithelial cells in response to Acinetobacter baumannii outer membrane protein A. FEMS Immunol Med Microbiol 54: 45-52.
106. Lee EY, Bang JY, Park GW, Choi DS, Kang JS, et al. (2007) Global proteomic profiling of native outer membrane vesicles derived from Escherichia coli. Proteomics 7: 3143-3153.

107. Choi CH, Lee JS, Lee YC, Park TI, Lee JC (2008) Acinetobacter baumannii invades epithelial cells and outer membrane protein $\mathrm{A}$ mediates interactions with epithelial cells. BMC Microbiol 8: 216.

108. Kwon SO, Gho YS, Lee JC, Kim SI (2009) Proteome analysis of outer membrane vesicles from a clinical Acinetobacter baumannii isolate. FEMS Microbiol Lett 297: 150-156.

109. Kieselbach T, Zijnge V, Granstrom E, Oscarsson (2015) J Proteomics of Aggregatibacter actinomycetemcomitans Outer Membrane Vesicles. PloS ONE 10: e0138591.

110. Choi DS, Kim DK, Choi SJ, Lee J, Choi JP, et al. (2011) Proteomic analysis of outer membrane vesicles derived from Pseudomonas aeruginosa. Proteomics 11: 3424-3429.

111. Mullaney E, Brown PA, Smith SM, Botting CH, Yamaoka YY, et al. (2009) Proteomic and functional characterization of the outer membrane vesicles from the gastric pathogen Helicobacter pylori. Proteomics Clin Appl 3: 785-796.

112. Jang KS, Sweredoski MJ, Graham RL, Hess S, Clemons WM (2014) Comprehensive proteomic profiling of outer membrane vesicles from Campylobacter jejuni. J Proteomics 98: 90-98.

113. Hellman J, Loiselle PM, Zanzot EM, Allaire JE, Tehan MM, et al. (2000) Release of gram-negative outer-membrane proteins into human serum and septic rat blood and their interactions with immunoglobulin in antiserum to Escherichia coli J5. J Infect Dis 181: 1034-1043.

114. Brandtzaeg P, Bryn K, Kierulf P, Ovstebo R, Namork E, et al. (1992) Meningococcal endotoxin in lethal septic shock plasma studied by gas chromatography, mass-spectrometry, ultracentrifugation, and electron microscopy. J Clin Invest 89: 816-823.

115. Mendez JA, Soares NC, Mateos J, Gayoso C, Rumbo C, et al. (2012) Extracellular proteome of a highly invasive multidrug-resistant clinical strain of Acinetobacter baumannii. J Proteome Res 11: 5678-5694.

116. Kahnt J, Aguiluz K, Koch J, Treuner-Lange A, Konovalova A, et al. (2010) Profiling the outer membrane proteome during growth and development of the social bacterium Myxococcus xanthus by selective biotinylation and analyses of outer membrane vesicles. J Proteome Res 9: 5197-5208.

117. Pierson T, Matrakas D, Taylor YU, Manyam G, Morozov VN, et al. (2011) Proteomic characterization and functional analysis of outer membrane vesicles of Francisella novicida suggests possible role in virulence and use as a vaccine. J Proteome Res 10: 954-967.

118. Jain S, Graham C, Graham RL, McMullan G, Ternan NG (2011) Quantitative proteomic analysis of the heat stress response in Clostridium difficile strain 630. J Proteome Res 10: 3880-3890.

119. Molloy MP, Herbert BR, Slade MB, Rabilloud T, Nouwens AS, et al. (2000) Proteomic analysis of the Escherichia coli outer membrane. Eur J Biochem 267: 2871-2881.

120. Tiwari V, Moganty RR (2013) Structural studies on New Delhi Metallobeta-lactamase (NDM-2) suggest old beta-lactam, penicillin to be better antibiotic for NDM-2-harbouring Acinetobacter baumanni. J Biomol Struct Dyn 31: 591-601.

121. Nwugo CC, Gaddy JA, Zimbler DL, Actis LA (2011) Deciphering the iron response in Acinetobacter baumannii: A proteomics approach. J Proteomics 74: 44-58.

122. Soares AJ, Santos MF, Trugilho MR, Neves-Ferreira AG, Perales J, et al. (2009) Differential proteomics of the plasma of individuals with sepsis caused by Acinetobacter baumannii. J Proteomics 73: 267-278.

123. Ciofu O, Beveridge TJ, Kadurugamuwa J, Walther-Rasmussen J, Hoiby (2000) N Chromosomal beta-lactamase is packaged into membrane vesicles and secreted from Pseudomonas aeruginosa. J Antimicrob Chemother 45: 9-13.

124. Tiwari V (2014) OMVproteomics to discover the pathogenicity of Acinetobacter baumannii. J Data Mining Genomics Proteomics 5: el16.

125. Bonnington KE, Kuehn MJ (2014) Protein selection and export via outer membrane vesicles. Biochim Biophys Acta 1843: 1612-1619. 
126. McCarthy Y, Dow JM, Ryan RP (2011) The Ax21 protein is a cell-cell signal that regulates virulence in the nosocomial pathogen Stenotrophomonas maltophilia. J Bacteriol 193: 6375-6378.

127. Ferrer-Navarro M, Planell R, Yero D, Mongiardini E, Torrent G, et al. (2013) Abundance of the Quorum-Sensing Factor Ax21 in Four Strains of Correlates with Mortality Rate in a New Zebrafish Model of Infection. PloS ONE 8: e67207.

128. Bahar O, Pruitt R, Luu DD, Schwessinger B, Daudi A, et al. (2014) The Xanthomonas Ax 21 protein is processed by the general secretory system and is secreted in association with outer membrane vesicles. Peer J 2: (e242).

129. Park HJ, Lee SW, Han SW (2014) Proteomic and functional analyses of a novel porin-like protein in Xanthomonas oryzae pv. oryzae. J Microbiol 52: 1030-1035.

130. Qian G, Zhou Y, Zhao Y, Song Z, Wang S, et al. (2013) Proteomic analysis reveals novel extracellular virulence-associated proteins and functions regulated by the diffusible signal factor (DSF) in Xanthomonas oryzae pv. oryzicola. J Proteome Res 12:3327-3341.

131. Ryan RP, Dow JM (2010) Communication with a growing family: diffusible signal factor (DSF) signaling in bacteria. Trend Microbiol 19: 145-152.

132. Fouhy Y, Scanlon K, Schouest K, Spillane C, Crossman L, et al. (2007) Diffusible signal factor-dependent cell-cell signaling and virulence in the nosocomial pathogen Stenotrophomonas maltophilia. J Bacteriol 189: 4964-4968.

133. Twomey KB, O'Connell OJ, McCarthy Y, Dow JM, O'Toole GA, et al. (2012) Bacterial cis-2-unsaturated fatty acids found in the cystic fibrosis airway modulate virulence and persistence of Pseudomonas aeruginosa. ISME J6: 939-950.

134. Minami A, Fujiwara M, Furuto A, Fukao Y, Yamashita T, et al. (2009) Alterations in detergent-resistant plasma membrane microdomains in Arabidopsis thaliana during cold acclimation. Plant Cell Physiol 50: 341-359.

135. Berlanda Scorza F, Doro F, Rodriguez-Ortega MJ, Stella M, Liberatori S, et al. (2008) Proteomics characterization of outer membrane vesicles from the extraintestinal pathogenic Escherichia coli DeltatolR IHE3034 mutant. Mol Cell Proteomics 7: 473-485.

136. Nevot M, Deroncele V, Messner P, Guinea J, Mercade E (2006) Characterization of outer membrane vesicles released by the psychrotolerant bacterium Pseudoalteromonas antarctica NF3. Env Microbiol 8: 1523-1533.

137. Ferrari G, Garaguso I, Adu-Bobie J, Doro F, Taddei AR, et al. (2006) Outer membrane vesicles from group B Neisseria meningitidis delta gna33 mutant: proteomic and immunological comparison with detergentderived outer membrane vesicles. Proteomics 6: 1856-1866.

138. Cabral MP, Soares NC, Aranda J, Parreira JR, Rumbo C, et al. (2011) Proteomic and functional analyses reveal a unique lifestyle for Acinetobacter baumannii biofilms and a key role for histidine metabolism. J Proteome Res 10: 3399-3417.

139. Peng X, Xu C, Ren H, Lin X, Wu L, et al. (2005) Proteomic analysis of the sarcosine-insoluble outer membrane fraction of Pseudomonas aeruginosa responding to ampicilin, kanamycin, and tetracycline resistance. J Proteome Res 4: 2257-2265.

140. Siroy A, Cosette P, Seyer D, Lemaitre-Guillier C, Vallenet D, et al. (2006) Global comparison of the membrane subproteomes between a multidrug resistant Acinetobacter baumannii strain and a reference strain. J Proteome Res 5: 3385-3398.

141. Marti S, Sanchez-Cespedes J, Oliveira E, Bellido D, Giralt E, et al. (2006) Proteomic analysis of a fraction enriched in cell envelope proteins of Acinetobacter baumannii. Proteomics 6: S82-S87.

142. Vashist J, Tiwari V, Kapil A, Rajeswari MR (2010) Quantitative profiling and identification of outer membrane proteins of beta-lactam resistant strain of Acinetobacter baumannii. J Proteome Res 9: 1121-1128.

143. Soares NC, Cabral MP, Parreira JR, Gayoso C, Barba MJ, et al. (2009) 2 $\mathrm{DE}$ analysis indicates that Acinetobacter baumannii displays a robust and versatile metabolism. Proteome Sci 7: 37.
144. Tiwari V, Vashistt J, Kapil A, Moganty RR (2012) Comparative proteomics of inner membrane fraction from carbapenem-resistant Acinetobacter baumannii with a reference strain. PloS ONE 7: e39451.

145. Mazzoli R, Fattori P, Lamberti C, Giuffrida MG, Zapponi M, et al. (2011) High isoelectric point sub-proteome analysis of Acinetobacter radioresistens $\mathrm{S} 13$ reveals envelope stress responses induced by aromatic compounds. Mol Bio Syst 7: 598-607.

146. Yun SH, Choi CW, Kwon SO, Park GW, Cho K, et al. (2011) Quantitative proteomic analysis of cell wall and plasma membrane fractions from multidrug-resistant Acinetobacter baumannii. J Proteome Res 10: $459-469$

147. Lee HY, Chen CL, Wang SB, Su LH, Chen SH, et al. (2011) Imipenem heteroresistance induced by imipenem in multidrug-resistant Acinetobacter baumannii: mechanism and clinical implications. Int J Antimicrob Agents 37: 302-308.

148. Zainal-Abidin Z, Veith PD, Dashper SG, Zhu Y, Catmull DV, et al. (2012) Differential proteomic analysis of a polymicrobial biofilm. J Proteome Res 11: 4449-4464

149. Szempruch AJ, Dennison L, Kieft R, Harrington JM, Hajduk SL (2016) Sending a message: extracellular vesicles of pathogenic protozoan parasites. Nat Rev Microbiol 14: 669-675.

150. Ogorevc E, Kralj-Iglic V, Veranic P (2013) The role of extracellular vesicles in phenotypic cancer transformation. Radiology and oncology 47: 197-205.

151. Gujrati V, Kim S, Kim SH, Min JJ, Choy HE, et al. (2014) Bioengineered bacterial outer membrane vesicles as cell-specific drug-delivery vehicles for cancer therapy. ACS Nano 8: 1525-1537.

152. Chitcholtan K, Hampton MB, Keenan JI (2008) Outer membrane vesicles enhance the carcinogenic potential of Helicobacter pylori. Carcinogenesis 29: 2400-2405

153. Muraoka S, Kume H, Watanabe S, Adachi J, Kuwano M, et al. (2012) Strategy for SRM-based verification of biomarker candidates discovered by iTRAQ method in limited breast cancer tissue samples. J Proteome Res 11: 4201-4210.

154. Durig A, Kouskoumvekaki I, Vejborg RM, Klemm P (2010) Chemoinformatics-assisted development of new anti-biofilm compounds. Appl Microbiol Biotechnol 87: 309-317.

155. Choi DS, Lee JM, Park GW, Lim HW, Bang JY, et al. (2007) Proteomic analysis of microvesicles derived from human colorectal cancer cells. J Proteome Res 6: 4646-4655.

156. Leong S, Nunez AC, Lin MZ, Crossett B, Christopherson RI, et al. (2012) iTRAQ-based proteomic profiling of breast cancer cell response to doxorubicin and TRAIL. J Proteome Res 11: 3561-3572.

157. Nie S, Lo A, Wu J, Zhu J, Tan Z, et al. (2014) Glycoprotein biomarker panel for pancreatic cancer discovered by quantitative proteomics analysis. J Proteome Res 13: 1873-1884.

158. Brown L, Wolf JM, Prados-Rosales R, Casadevall A (2015) Through the wall: extracellular vesicles in Gram-positive bacteria, mycobacteria and fungi. Nat Rev Microbiol 13: 620-630.

159. Aguilera L, Toloza L, Gimenez R, Odena A, Oliveira E, et al. (2014) Proteomic analysis of outer membrane vesicles from the probiotic strain Escherichia coli Nissle. Proteomics 14: 222-229.

160. Rodriguez-Ortega MJ, Norais N, Bensi G, Liberatori S, Capo S, et al. (2006) Characterization and identification of vaccine candidate proteins through analysis of the group A Streptococcus surface proteome. Nature Biotechnol 24: 191-197.

161. Delgado M, Yero D, Niebla O, Gonzalez S, Climent Y, et al. (2007) Lipoprotein NMB0928 from Neisseria meningitidis serogroup B as a novel vaccine candidate. Vaccine 25: 8420 -8431.

162. Tiwari V, Roy R, Tiwari M (2015) Antimicrobial active herbal compounds against Acinetobacter baumannii and other pathogens. Front Microbiol 6: 618.

163. Tiwari V (2014) Anti-bacterial Activity of Polyvinyl Pyrrolidone Capped Silver Nanoparticles on the Carbapenem Resistant Strain of Acinetobacter baumannii. J Nanomed Nanotechnol 5: 2-6. 